P68

A STUDY TO INVESTIGATE THE CLINICAL USE AND OUTCOMES OF EZPAP POSITIVE PRESSURE DEVICE

doi:10.1136/thoraxjn-2011-201054c.68

S Elliott. Medway Maritime Hospital, Gillingham, UK

Introduction The EZPAP is a positive pressure hand held device that amplifies an input flow of either air or oxygen approximately four times and so positive pressure is maintained throughout the patients breathing cycle. This augmentation provides a larger flow and volume with less effort than an unsupported inspiration and PEP is provided on expiration. Studies have shown that it is easier to tolerate than Intermittent Positive Pressure Breathing (IPPB) and has greater effect in reversing atelectasis than incentive spirometry. Thus, can be used within physiotherapy to increase lung volume, clear secretions and improve gaseous exchange.

Objectives To measure clinical outcomes of the EZPAP in relation to increasing lung volume, sputum clearance and gaseous exchange.

Method Data were collected from 20 patients identified as suitable for EZPAP interventions and physiological observations pretreatment and posttreatment were recorded, additionally physiotherapists and patients were asked to comment on the treatment session.

Results Patients who demonstrated decreased lung volume showed an $82 \%$ increase of air entry on auscultation and a $72 \%$ increase in thoracic expansion, whereas audible crepitations on auscultation reduced by $45 \%$ and $12 \%$ of patients demonstrated a more effective cough to clear secretions. With respect to gas exchange, there was a $33 \%$ increase in $\mathrm{SaO} 2$ and $12 \%$ of patients were weaned from oxygen post intervention. Additionally, this study yielded results to suggest that EZPAP also reduces the work of breathing with a mean decrease of respiratory rate of 3.5 breaths per minute. Both physiotherapists and patients found the device easy to use with a high level of compliance. Improvements in the patients were also rapid with an average of three sessions across 1.2 days.

Conclusions It can be concluded from this study that the EZPAP positive pressure device is an extremely versatile tool for the physiotherapist at a district general hospital in the management of all respiratory problems with a high level of patient compliance, ease of use and rapid clinical improvements.

Recommendations Further randomised control trials need to be undertaken utilising radiological examination and arterial blood gas sampling to avoid bias in physiological observation measurement.

\section{P69 A STUDY OF THE PREVALENCE OF HYPOXAEMIA, HYPERCAPNIA, HYPEROXAEMIA AND ACIDOSIS IN HOSPITAL BLOOD GAS SPECIMENS (WITH CLINICAL OUTCOMES)}

doi:10.1136/thoraxjnl-2011-201054c.69

F Simpson, B R O'Driscoll. Salford Royal University Hospital, Salford, UK

Introduction Some clinicians take an aggressive approach to supplemental oxygen therapy to avoid the dangers of hypoxaemia but many patients, especially those with COPD, are at risk from uncontrolled oxygen therapy. Furthermore, recent publications have suggested an independent association between hyperoxaemia and inhospital mortality of ICU patients (de Jonge 2008, Kilgannon 2010). Methods We studied a 1-year database of 29587 hospital blood gas specimens including samples from A\&E and ICU. Only the first adequate sample from each patient was analysed; $(n=7956)$. Samples were grouped in saturation bands corresponding to the recommended target saturation ranges in the BTS Emergency Oxygen Guideline and saturation bands above, below and between these ranges (Abstract P69 table 1). A random sub-sample of 360 specimens was analysed in more detail for clinical outcomes and the prevalence of risk factors for type 2 respiratory failure.
Abstract P69 Table 1

\begin{tabular}{lclcc}
\hline $\begin{array}{l}\text { Saturation } \\
\text { range }\end{array}$ & Number (\%) & $\begin{array}{l}\text { Percent } \\
\text { hypercapnic }\end{array}$ & $\begin{array}{l}\text { Respiratory } \\
\text { acidosis }\end{array}$ & $\begin{array}{l}\text { Metabolic } \\
\text { acidosis }\end{array}$ \\
\hline$>98 \%$ & $2077(26 \%)$ & $16 \%$ & $5 \%$ & $10 \%$ \\
$94-98 \%$ & $2530(32 \%)$ & $16 \%$ & $5 \%$ & $8 \%$ \\
$92-94 \%$ & $634(8 \%)$ & $22 \%$ & $5 \%$ & $8 \%$ \\
$88-92 \%$ & $744(9 \%)$ & $25 \%$ & $7 \%$ & $8 \%$ \\
$<88 \%$ & $1950(24 \%)$ & $36 \%$ & $11 \%$ & $11 \%$ \\
Total & $\mathbf{7 9 6 5 ( 1 0 0 \% )}$ & $\mathbf{2 2 \%}$ & $\mathbf{7 \%}$ & $\mathbf{9 \%}$ \\
\hline
\end{tabular}

Results Excluding A\&E cases, only $10 \%$ of blood gas samples demonstrated Type 1 respiratory failure with $\mathrm{PO}_{2}<8 \mathrm{kPa}(60 \mathrm{~mm}$ $\mathrm{Hg}$ ) and normal $\mathrm{CO}_{2}$ levels. Thirty per cent of first $\mathrm{A} \& \mathrm{E}$ samples had Type 1 respiratory failure. Of 7965 samples analysed, 22\% were hypercapnic with $\mathrm{PCO}_{2}>6 \mathrm{kPa}(45 \mathrm{~mm} \mathrm{Hg}$ ) consistent with Type 2 respiratory failure and the oxygen saturation was above $92 \%$ in $54 \%$ of these hypercapnic patients. $22 \%$ of all samples were hyperoxaemic with $\mathrm{PO}_{2}>15 \mathrm{kPa}(112 \mathrm{~mm} \mathrm{Hg})$ and $26 \%$ had saturation $>98 \%$. Within the sub-sample of 360 specimens there were 72 inhospital deaths (20\%). The lowest mortality was $8 \%$ in the $88-92 \%$ saturation range and the highest mortality was 39\% in those with saturation $<88 \%$. Of 85 patients documented to be at risk of Type 2 respiratory failure, $62(73 \%)$ had oxygen saturations greater than the 88-92\% target range recommended in BTS Guidelines.

Conclusions Despite the introduction of the BTS Guidelines, hyperoxaemia is still a common finding in blood gas specimens in 2011. Type 2 respiratory failure is twice as common as Type 1 failure except for $A \& E$ samples but the majority of patients at risk of Type 2 respiratory failure had oxygen saturations above the recommended target range. These results demonstrate the need for a prospective study of the link between oxygen therapy and overall hospital mortality.

\section{P70 DOES MEETING THE CLINICAL CRITERIA FOR THE SYSTEMIC INFLAMMATORY RESPONSE SYNDROME EQUATE TO BIOCHEMICAL INFLAMMATION FOLLOWING CARDIAC SURGERY?}

doi:10.1136/thoraxjnl-2011-201054c.70

F M Conway, S E Gordon, G J Quinlan, T W Evans, N S MacCallum. Unit of Critical Care, Biomedical Research Unit, Imperial College, Royal Brompton Hospital, London, UK

Introduction and Objectives The systemic inflammatory response syndrome (SIRS) criteria were developed in part as entry criteria for clinical trials of generalised inflammation from various causes. Despite being commonly used to assess inflammation in cardiac surgery, no one has evaluated whether the clinical defining criteria for inflammation equate to biochemical inflammation in this patient population. Our aim was to investigate whether the SIRS criteria equate to established biochemical indices of inflammation following cardiac surgery.

Methods Retrospective analysis of prospectively collected data from an adult intensive care unit of a specialist cardiothoracic centre in the UK. 93 adult patients undergoing cardiac surgery admitted over a 34-month period between March 2004 and January 2007 were included. Patients were scored on the SIRS criteria from before to $72 \mathrm{~h}$ post-surgery. ELISAs were performed on archived plasma samples taken before surgery and at 4, 24, 48 and $72 \mathrm{~h}$ post-operatively to determine concentrations of IL-1ß, IL-1RA, IL-6, IL-8, IL10, myeloperoxidase (MPO) and $\mathrm{C}$ reactive protein (CRP).

Results Significantly elevated levels of all inflammatory indices were detected post-operatively with the exception of IL-1B. Patients meeting SIRS criteria demonstrated higher levels of IL-6, IL-1RA and CRP compared to those who did not meet the criteria. An absence or only weak correlation was found between SIRS and mass of biomarker. 